\title{
A commentary on Manzano-Bort et al. (2021) 'Experience of mental health nurses regarding mechanical restraint in patients with psychomotor agitation: A qualitative study'
}

Dear Editor,

I read, with great interest, the article 'Experience of mental health nurses regarding mechanical restraint in patients with psychomotor agitation: A qualitative study' by Manzano-Bort et al. (2021). The authors interviewed mental health nurses in order to understand their experience when managing patients with psychomotor agitation and the factors that lead to the use of mechanical restraints (MR).

Reducing the use of coercive measures has been an objective in psychiatric care for many years (Gooding et al., 2020). In addition, psychiatry has also been frequently criticised for 'labelling' and 'categorising' people into 'disorders', thereby dehumanising and marginalising people, without adequately considering their personal experiences, culture, ethnicity, religion or even past traumas. Currently, person-centred mental health care aims to counter that tendency and transform care according to the following principles: a holistic or integrative care that responds to a person's individual needs and values; treating each person with dignity, respect and compassion; and empowering patients as partners involved in decision-making. Thus, I welcome the authors' contribution towards implementing positive strategies to further reduce or eliminate the application of MR in users with psychomotor agitation.

Given the potential aggressive or violent behaviour by a patient, several intervention strategies should be considered: a) preventive strategies (to avoid potential aggression), including direct observation, self-awareness, therapeutic education and assertiveness training; b) anticipatory strategies (to decrease potential aggression), including de-escalation techniques, environmental modifications, rapid reassurance behavioural interventions, and finally, the patient medication adherence. Only after exhausting preventive and anticipatory measures should the use of containment measures be considered. In this case, one should consider the risks (physical, psychological, ethical and legal) of different types of MR, and always opt for the least restrictive (Pariseau-Legault et al., 2019). It is recommended that nurses following some commitments: 1) apply MR only after exhausting preventive measures; 2) if MR becomes necessary, obtain consent, when possible from the patient, or alternatively from the patient's family or significant other; 3) explain what will be done and why to the patient; 4) adjust the containment measures to the patient's situation; 5) comply with the manufacturer's instructions when applying the restraint mechanism; 6) during application of the MR, monitor the patient as often as their condition dictates; 7) reassess the need for the MR and the possibility of its replacement with a less restrictive measure; 8) remove the containment measure as soon as possible; and finally, 9) record nursing procedures in the clinical file.

An important issue, not fully explored by the authors, is how insufficient professional training can promote the use of coercive methods. The literature highlights that training and education of professionals are associated with positive changes in their attitudes, knowledge and trust, thus leading to a decrease in the severity of aggressive incidents by patients and, consequently, a reduction in the use of coercive measures (Maagerø-Bangstad et al., 2020). Training is also required to develop advanced care planning, conduct debriefings with affected patients after application of coercive measures, and properly document the process (Steinert et al., 2020).

Healthcare organisations should be responsible for ensuring the timely recognition and prevention of disruptive behaviours and the quality of therapeutic management of patients with such behaviours. The importance of risk assessment-with the Brøset Violence Checklist (BVC) or another instrument-and stratification (often justifying the imposition of coercive measures), as a simple and rapid intervention, should be integrated into clinical practice, allowing for a reduction in incidence and severity of aggressive behaviour (Steinert et al., 2020; Verbeke et al., 2019). Clinical intervention and research can produce knowledge that will promote the implementation of evidence- and consensus-based guidelines to reduce coercion in mental health care.

I hope this commentary contributes to a discussion on the role of nurses in reducing restrictive measures, based on a patient-centred care approach. Difficulties and threats can be transformed into strengths and opportunities, given the discoveries and challenges contributing to the recognition and defence of human rights in a psychiatric context.

\section{FUNDING INFORMATION}

This work is funded by national funds through FCT-Fundação para a Ciência e a Tecnologia, I.P. (UIDB/05704/2020 and UIDP/05704/2020) and under the Scientific Employment StimulusInstitutional Call-[CEECINST/00051/2018].

\section{CONFLICT OF INTEREST}

The author declared no conflicts of interest with respect to the authorship and/or publication of this article. 
Carlos Laranjeira PhD, MSc, MHN, Professor ${ }^{1,2,3}$ iD

${ }^{1}$ School of Health Sciences, Polytechnic of Leiria, Leiria, Portugal

${ }^{2} \mathrm{RECl}$ (Research in Education and Community Intervention),

Piaget Institute, Viseu, Portugal

${ }^{3}$ Center for Innovative Care and Health Technology (ciTechCare),

Polytechnic of Leiria, Leiria, Portugal

Correspondence

Carlos Laranjeira, School of Health Sciences of Polytechnic

of Leiria, Campus 2, Morro do Lena, Alto do Vieiro, Apartado

4137, 2411-901 Leiria Leiria, Portugal.

Email: carlos.laranjeira@ipleiria.pt

\section{ORCID}

Carlos Laranjeira (D) https://orcid.org/0000-0003-1080-9535

\section{REFERENCES}

Gooding, P., McSherry, B., \& Roper, C. (2020). Preventing and reducing 'coercion' in mental health services: An international scoping review of English-language studies. Acta Psychiatrica Scandinavica, 142(1), 27-39. https://doi.org/10.1111/acps.13152
Maagerø-Bangstad, E. R., Sælør, K. T., Lillevik, O. G., \& Ness, O. (2020). Exploring staff conceptions of prevention and management practices in encounters with staff-directed aggression in supported housing following education and training. International Journal of Mental Health Systems, 14, 60. https://doi.org/10.1186/s13033020-00387-2

Manzano-Bort, Y., Mir-Abellán, R., Via-Clavero, G., Llopis-Cañameras, J., Escuté-Amat, M., \& Falcó-Pegueroles, A. (2021). Experience of mental health nurses regarding mechanical restraint in patients with psychomotor agitation: A qualitative study. Journal of Clinical Nursing. https://doi.org/10.1111/jocn.16027. Epub ahead of print].

Pariseau-Legault, P., Vallée-Ouimet, S., Goulet, M.-H., \& Jacob, J.-D. (2019). Nurses' perspectives on human rights when coercion is used in psychiatry: A systematic review protocol of qualitative evidence. Systematic Reviews, 8, 318. https://doi.org/10.1186/s1364 3-019-1224-0

Steinert, T., Bechdolf, A., Mahler, L., Muche, R., Baumgardt, J., BühlingSchindowski, F., Cole, C., Kampmann, M., Sauter, D., Vandamme, A., Weinmann, S., \& Hirsch, S. (2020). Implementation of guidelines on prevention of coercion and violence (PreVCo) in psychiatry: Study protocol of a randomized controlled trial (RCT). Frontiers in Psychiatry, 11, 579176. https://doi.org/10.3389/fpsyt.2020.579176

Verbeke, E., Vanheule, S., Cauwe, J., Truijens, F., \& Froyen, B. (2019). Coercion and power in psychiatry: A qualitative study with expatients. Social Science \& Medicine, 223, 89-96. https://doi. org/10.1016/j.socscimed.2019.01.031 